\title{
Lung auscultation - Identification of common lung sound abnormalities and associated pathologies
}

\author{
Najee Boucher ${ }^{1}$, Andrzej Prystupa ${ }^{2}$, Agnieszka Witczak², Ewa Walczak³ , Grzegorz Dzida², \\ Lech Panasiuk ${ }^{3}$ \\ ${ }^{1}$ Student Scientific Society, Medical University, Lublin, Poland \\ ${ }^{2}$ Department of Internal Medicine, Medical University, Lublin, Poland \\ ${ }^{3}$ Department of Family Medicine, Institute of Rural Health, Lublin, Poland
}

Boucher N, Prystupa A, Witczak A, Walczak E Dzida G, Panasiuk L. Lung auscultation - Identification of common lung sound abnormalities and associated pathologies. J Pre-Clin Clin Res. 2013; 7(1): 32-35.

\section{Abstract}

Objective: The purpose of this review is to show the present state of art on breath sounds and lung auscultation, including normal and adventitious lung sounds, especially how they originate, and to which of the most common diseases they are related.

Methods: The history of auscultation and the first stethoscope is presented, followed by the classification and description of physiologic lung sounds with regard to the lung regions where they should be heard and the sound characteristics. Added lung sounds, including their classification, distinction between continuous and discontinuous sound, as well as the presentation of the diseases most commonly associated with them are presented.

Results: Since it may be found that breath sounds are difficult to understand it is reasonable to present a simple method of lung auscultation that may be helpful in the education of medical students. The usefulness of lung auscultation in diagnosing the most common diseases, with special regard to lung disorders, is of great importance.

Discussion/Conclusion: The short and simple presentation of lung sound, auscultation methods and their application in clinical practice has been presented, and may be helpful for medical staff.

\section{Key words}

lung auscultation, continuous sounds, discontinuous sounds, stethoscope, asthma, bronchitis, wheezes, rhonchi, crackles, pneumonia, emphysema

\section{INTRODUCTION}

Lung auscultation is a significant part of physical examination which is helpful in diagnosing respiratory disorders. Proper understanding of the physiology and pathology of lung sounds is necessary for applying lung auscultation with good effect, including the choice of further diagnostic steps and preliminary treatment, as well as prognosis estimation.

\section{MATERIALS AND METHODS}

The stethoscope is a helpful tool in the evaluation and differential diagnosis of cardiac and pulmonary disorders. Its use and application can aid the physician tremendously. The skills necessary to translate auscultation findings into an accurate diagnosis of the disease require both knowledge and understanding of the physiology behind the sounds, and a great deal of experience. Understanding of the location, duration and features of sounds heard over the lungs all play an intricate role in establishing a differential diagnosis. Presented is a short review on the physiology behind lung sounds and an outline of diagnostic steps with the use of a stethoscope that may be helpful in differential diagnosing of pulmonary disorders.

Address for correspondence: Andrzej Prystupa, Chair and Department of Internal Medicine, Medical University, Staszica 16, 20-081 Lublin, Poland

e-mail: aprystup@mp.pl

Received: 12 October 2012; accepted: 11 June 2013

\section{HISTORY}

Auscultation is one of the steps included in physical examination. It is the method of listening to the sounds of the body and distinguishing between sounds that are physiologic and sounds that are pathologic. Primarily, auscultation is used to evaluate heart, pulmonary and gastrointestinal sounds. The history of auscultation dates back to Hypocrites who directly placed his ear on a patient's chest to hear the sounds [1]. This method was used for thousands of years, until the nineteenth century when a French physician, Rene Theophile Hyacinthe Laennec, invented the stethoscope [2]. He initially created a cylinder with paper and later improved on his invention with two hollow cylindrical pieces of wood connected to one another, and a cone-shaped attachment containing a brass cylinder [2]. He used the attached conical piece during heart auscultation and removed it when he needed to auscultate the lungs. He named this instrument a 'stethoscope' [2], the name originating from the Greek words 'stethos' and 'scope', which refer to the chest and to the view, respectively [1].

Over time, the stethoscope has evolved into the device presently in use, including electrical stethoscopes that can make recordings and amplify the sound more than conventional stethoscopes. Even with this new technology, the basics of the stethoscope have remained the same since its invention in the nineteenth century (Fig. 1). 


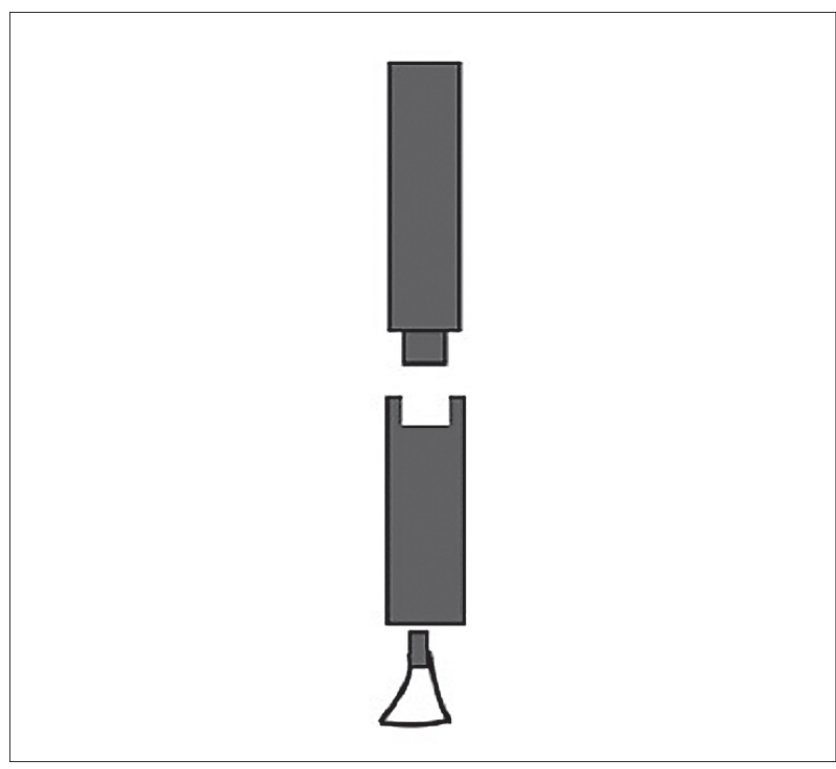

Figure 1. Laennec's first stethoscope.

\section{NORMAL LUNG SOUNDS}

The first known description of lung sounds was given by Laennec who invented the first stethoscope [2]. However, it was not until much later that physicians were able to discern the different physiologic and pathologic lung sounds that are found today. It is also important to note that during heart auscultation the observer hears uniformity from one beat to the next. However, this is not the case in lung auscultation where uniformity is not heard from breath to breath [3].

Lung sounds are created due to turbulence of the inspired air in the airways. As air rushes in on inspiration, it creates a turbulent flow as the air presses against the respiratory walls and does not become a laminar flow until the distal airways [4]. Furthermore, Forgacs demonstrated that distal to the lobar or segmental bronchi, the velocity becomes too low and does not cause turbulence thereby becoming silent $[4,5]$. This has led to the speculation that lung sounds are produced by the glottis, the friction of the inspired gasses on the airways, and the flow of gasses from airways of different diameters during respiration [3,5]. The lung sounds are then 'transmitted to the chest wall via airways or the lung parenchyma' [5], and can be heard when the stethoscope is placed on the chest wall.

The region where the stethoscope is placed on the chest wall depicts which part of the respiratory system is to be evaluated. There are four locations: tracheal, bronchial, bronchovesicular, and vesicular [6]. Tracheal breath sounds can be heard when the stethoscope is placed over the trachea. Bronchial breath sounds can be heard anteriorly when the stethoscope is placed over the manubrium and posteriorly when it is placed between the C7 and T3 vertebrae [6]. Bronchovesicular breath sounds can be heard anteriorly when the stethoscope is in the intercostal spaces of the first and second ribs, and posteriorly when placed lateral to the T3 and T5 vertebrae [7]. Vesicular breath sounds can be heard peripherally throughout the lung in the intercostal spaces from apex to base, and lateral to the bronchovesicular sounds. It is also important to note that lung sounds cannot be heard over the scapula [6].
It is important to understand the characteristics of lung sounds so that normal physiologic sounds can be distinguished from pathologic sounds. Tracheal sounds tend to be loud, harsh blowing and high-pitched while being heard equally between inspiration and expiration [6]. Bronchial sounds are different, with the expiratory phase heard for much longer than the inspiratory phase. The sound is loud and has a high-pitched blowing characteristic [6]. Bronchovesicular sounds, like tracheal sounds, can be heard equally throughout inspiration and expiration. The sound is lower and has a lower pitch, described by Kirton as of 'breezy quality' [6]. Vesicular sounds can be heard much longer during inspiration and are low-pitched having a soft quality.

This review will focus on the pathologic lung sounds heard during auscultation and the associated diseases. Pathologic lung sounds are subdivided into discontinuous and continuous lung sounds (Fig. 2).

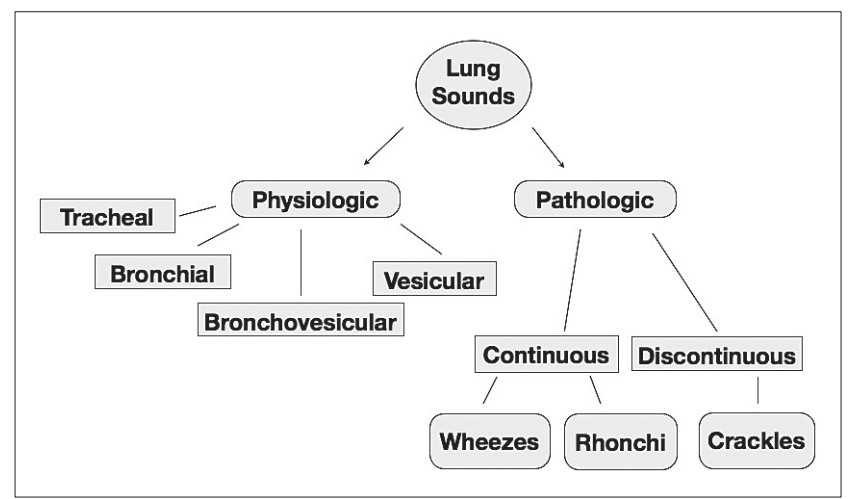

Figure 2. Classification of lung sounds.

\section{DISCONTINUOUS LUNG SOUNDS}

Discontinuous lung sounds are intermittent short-lived sounds that can be heard during inspiration and/or during expiration, and are called 'crackles' $[4,8]$. The presence and amount of crackles heard on auscultation coincide with the degree of severity of the disease [9]. Crackles are subdivided into inspiratory and expiratory; inspiratory crackles originate during inspiration when the pressure behind a closed airway becomes so great that it opens the abnormally closed airway, whereas expiratory crackles develop when the pressure inside the airway is once again decreased, causing the airway to close suddenly [10]. Both types tend to occur in patients who suffer chronic bronchitis, pulmonary fibrosis, congestive heart failure and pneumonia [7].

\section{DISEASES WITH CHARACTERISTIC DISCONTINUOUS LUNG SOUNDS}

Chronic obstructive bronchitis. Chronic obstructive bronchitis is a type of chronic obstructive pulmonary disease most commonly diagnosed in smokers [11]. It is characterized by inflammation of the airways resulting in productive cough due to excessive mucus production [11]. In chronic bronchitis, crackles can be heard due to collapse of lobar bronchi, and can be heard centrally during the end of expiration [7]. 
Pulmonary fibrosis. Pulmonary fibrosis is a chronic disease where the lung progressively consists of scar tissue and is characterized by a dry cough and breathlessness upon exercise [12]. Crackles can be heard peripherally and occur continuously during mid-to-late inspiration [13]. Early in the course of the disease, crackles can be heard at the lung bases; however, as pulmonary fibrosis progresses, crackles can also be heard over the upper areas of the lung [7].

Congestive heart failure. Congestive heart failure (CHF) usually develops due to impaired left heart function, resulting in the back-up of blood into the lungs and subsequent venous pulmonary hypertension that affects the right heart leading to peripheral congestion. In the course of $\mathrm{CHF}$, the crackles heard are due to the opening of previously compressed airways which is the result of the constrictive nature of the pulmonary oedema induced by the high end-diastolic left ventricular pressure. The crackles can be heard during late inspiration and rarely expiration, and classified as discontinuous crackles [3]. The location of the crackles is typically at the base of the lungs in upright body position or, in general, over the dependant regions, due to the gravitational force on the oedematous fluid. Together with the progression of CHF, crackles can be heard throughout the lung [7]. On the other hand, with effective treatment and subsequent improvement of the left heart function, the crackles rapidly dissipate [7].

Pneumonia. Pneumonia is a common disease caused by many pathogens. The intricate nature of pneumonia is different from other diseases causing discontinuous lung sounds. As described by Piirilä and Sovijärvi [5], in acute pneumonia, oedema and inflammatory cells create crackles by narrowing of the bronchi. As the pneumonia resolves, oedema clears and inflammatory cells accumulate. The crackles in acute pneumonia are described as mid- inspiratory crackles, compared to short end-inspiratory crackles in a patient with resolving pneumonia [7].

The timing and location of discontinuous lung sounds can seen in Table 1 below.

Table 1. Timing and location of discontinuous lung sounds.

\begin{tabular}{lll}
\hline Disease & $\begin{array}{l}\text { When can the crackles } \\
\text { be heard }\end{array}$ & $\begin{array}{l}\text { Where can the crackles } \\
\text { be auscultated on chest }\end{array}$ \\
\hline Chronic Bronchitis & Expiration & Centrally \\
\hline Pulmonary Fibrosis & Mid-Late Inspiration & Peripherally \\
\hline Congestive Heart Failure & Late Inspiration & Base of the Lungs \\
\hline Lobar Pneumonia & Mid Inspiration & Entire Affected Lobe \\
\hline
\end{tabular}

\section{CONTINUOUS LUNG SOUNDS}

Continuous sounds are subdivided into wheezes and rhonchi.

Wheezes. Wheezes are continuous high-pitched sounds with a frequency of $400 \mathrm{~Hz}$ or higher [14]. They are thought to come from vibrations of the walls of the airway at either one or multiple sites in the lung [3]. Wheezes can be heard without a stethoscope via the open mouth of the patient or with a stethoscope placed over the larynx in asthma patients, or over other lung fields in other diseases [14]. Murphy describes wheezes as occurring 'when air passing through a narrowed airway at high velocity produces a decrease in the gas pressure in the airway at the region of constriction' [3]. Wheezes are most commonly clinical signs of asthma, but can also occur in other diseases associated with obstructive airways [14].

Rhonchi. Rhonchi are continuous low-pitched lung sounds with a dominant frequency of $200 \mathrm{~Hz}$ or less [14]. Rhonchi are mostly heard when there is bronchial obstruction, especially with secretions [14].

\section{DISEASES WITH CHARACTERISTIC CONTINUOUS LUNG SOUNDS}

Asthma. Asthma is a chronic disease characterized by airway inflammation thereby limiting airflow in the airways [15]. Wheezes are the lung sounds most commonly heard in asthma patients. They are most commonly heard during expiration due to the air spaces being normally narrowed in that phase of respiration [15]. In order to hear the wheezes, the physician preferentially places the stethoscope directly over the trachea instead of the lung to hear the wheezes easier [14].

Emphysema. Emphysema, like chronic obstructive bronchitis, is a type of chronic obstructive pulmonary disease (COPD). It tends to affect smokers more commonly than non-smokers and develops within the apices of the lungs [16]. Emphysema is characterized by damage to the small bronchioles and alveoli, resulting in diminished vesicular sound as well as obstruction of the small airways. This leads to a decrease in the force necessary to expire air from the lungs [16]. The adventitious lung sounds associated with COPD, like emphysema, are wheezes [17].

\section{CONCLUSIONS}

Although much has changed in science since the first stethoscope was made by Laennec, auscultation is still an important diagnostic tool. Technology has greatly improved over the years, enabling the physician to enhance the quality of lung sounds heard on auscultation, and even make recordings with their stethoscopes. However, even with the technological advances, it is still important to understand the physiology behind lung sounds and the certain sounds associated with pulmonary pathologies. The presented study has provided a clear and concise introduction to the various lung sounds.

\section{REFERENCES}

1. Abdulla R. The history of the stethoscope. Pediatr Cardiol. 2001; 22(5): 371-372.

2. Roguin A. Rene Theophile Hyacinthe Laënnec (1781-1826): The Man Behind the Stethoscope. Clin Med Res. 2006; 4(3): 230-235.

3. Murphy R. Auscultation of the lung: past lessons, future possibilities. Thorax. 1981; 36(2): 99-107.

4. Forgacs P. Crackles and wheezes. Lancet 1967; 22: 203-205.

5. Jones A. A Brief Overview of the Analysis of Lung Sounds. Physiotherapy. 1995; 81: 37-42.

6. Kirton C. Physical assessment. Assessing breath sounds. Nursing. 1996; 26(6): 50-51.

7.Piirilä P, Sovijärvi AR.Crackles: recording, analysis and clinical significance. Eur Respir J. 1995; 8(12): 2139-48. 
8. Nath A, Capel L. Inspiratory crackles and mechanical events of breathing. Thorax. 1974; 29(6): 695-698.

9. Vyshedskiy A, Ishikawa S, Murphy RL Jr. Crackle pitch and rate do not vary significantly during a single automated-auscultation session in patients with pneumonia, congestive heart failure, or interstitial pulmonary fibrosis. Respir Care. 2011; 56(6): 806-817.

10. Vyshedskiy A, Alhashem R, Paciej R, Ebril, M, Rudman I, Fredberg J, Murphy R. Mechanism of inspiratory and expiratory crackles. Chest. 2009; 135(1): 156-164

11. Albertson T, Louie S, Chan A. The diagnosis and treatment of elderly patients with acute exacerbation of chronic obstructive pulmonary disease and chronic bronchitis. J Am Geriatr Soc. 2010; 58(3): 570-579.

12. Meltzer EB, Noble P W. Idiopathic pulmonary fibrosis. Diseases. Orphanet J Rare Dis. 2008; 3: 1750-1172.
13. Dalmasso F, Guarene M, Spagnolo R, Benedetto G, Righini G. A computer system for timing and acoustical analysis of crackles: a study in cryptogenic fibrosing alveolitis. Bull Eur Physiopathol Respir. 1984; 20(2): 139-144.

14. Meslier, N, Charbonneau, G, Racineux, J. Wheezes. Eur Respir J. 1995; 8(11): 1942-1948.

15. Habukawa C, Nagasaka Y, Murakami K, Takemura T. High-pitched breath sounds indicate airflow limitation in asymptomatic asthmatic children. Respirology 2009; 14(3): 399-403.

16. Omori H, Fujimoto K, Katoh, T. Computed-tomography findings of emphysema: correlation with spirometric values. Curr Opin Pulm Med. 2008; 14(2): 110-114.

17. Taplidou, SA, Hadjileontiadis, LJ. Wheeze detection based on timefrequency analysis of breath sounds. Comput Biol Med. 2007; 37(8): 1073-1083. 